https://www.journal-imab-bg.org

Original article

\title{
DIMENSIONAL RIDGE ALTERATIONS AND HISTOMORPHOMETRIC ANALYSIS FOLLOWING SOCKET PRESERVATION WITH PRF OR ALLOGRAFT. RANDOMIZED CONTROLLED CLINICAL STUDY
}

\author{
Vasilena Ivanova ${ }^{1}$, Ivan Chenchev ${ }^{1}$, Stefan Zlatev ${ }^{2}$, Nikolay Kanazirski ${ }^{1}$ \\ 1) Department of Oral Surgery, Faculty of Dental Medicine, Medical University \\ - Plovdiv, Bulgaria \\ 2) Department of Prosthetic dental medicine, Faculty of Dental Medicine, \\ Medical University - Plovdiv, Bulgaria.
}

\begin{abstract}
:
Purpose: The aim of this randomized controlled clinical trial was to evaluate the efficacy of A-PRF alone or in combination with freeze-dried bone allograft in improving vital bone formation and decreasing alveolar bone resorption.

Materials and methods: The study included 60 patients with 63 post-extraction sockets. They were divided into three groups - socket preservation with freeze-dried bone allograft (BoneAlbumin ${ }^{\mathrm{TM}}$, OrthoSera Dental, Gyor, Hungary), which consisted of 28 patients. The second group presented post-extraction sockets filled with PRF as a sole grafting material -23 patients and the third group was the control group - 12 patients. Two scans with Trios intraoral scanner were performed on each patient - one during the socket preservation procedure and one 4 months after. The changes of alveolar height and width were assessed on the superimposed virtual models obtained from the intraoral scanner. Bone biopsy was harvested from the preserved area with a trephine drill 4 months after the extraction.
\end{abstract}

Results: Results reveal that vertical and horizontal bone resorption were less pronounced in both of the tested groups as opposed in the control group, where these values were significantly higher. There was a significant difference between the quantity of the newly formed bone in the control group and both of the tested groups - PRF and allograft.

Conclusions: The study reveals that A-PRF as a sole grafting material or FDBA in combination with PRF membrane are both suitable for ridge preservation procedures. The study demonstrates a novel in vivo method for measuring bone resorption after ridge augmentation procedures.

Keywords: socket preservation, dental implants, allograft, PRF, Trios intraoral scanner, CAD/CAM,

\section{INTRODUCTION:}

Preserving the original volume and architecture of the alveolar bone after a tooth extraction is crucial in obtaining esthetic and functional prosthetic reconstruction after implant therapy. [1] After tooth loss, the alveolar ridge undergoes dimensional alterations which lead to a reduction of alveolar ridge height and width. [2] Clinical studies demonstrate an average of 0.7-1.5 mm of vertical and 4.0-4.5 mm of horizontal bone resorption 6 months after tooth extraction without socket preservation. [3] In the long term, reduced alveolar bone volume and subsequent soft tissue collapse result in loss of function, the impossibility for placing dental implants, and difficulty in conventional prosthetic treatment. [4] A variety of surgical techniques and grafting materials are applied in socket preservation procedure. The materials used are different in composition and source, but one of the most important factors is the possibility of their being resorbed and substituted by newly formed bone. [5]

PRF is a second-generation platelet concentrate that contains a high concentration of inactive, functional, intact platelets contained in a fibrin matrix. [6] The scientific justification for the use of platelet concentrates is based on the fact that platelets serve as a reservoir for many growth factors, which play an important role in the healing process of both soft and hard tissues. PRF stimulates osteoblastic proliferation, and histological data in the literature show that it has an effect on neoangiogenesis. [7]

FDBA is an osteoconductive material that functions as a scaffold to induce new bone formation. [8] FDBA has been proven to be a successful bone substitute in cases where conserving the alveolar is intended to conserve bone volume and form new bone.[9] Before the mineralized FDBA is able to express its osteoinductivity, it must undergo an osteoclastic resorption phase in order to degrade the mineral content and release bone morphogenic proteins (BMPs) [10]. The osteoconductive properties of FDBA are more pronounced than those of DFDBA [5]. 


\section{MATERIALS AND METHODS}

The research protocol of this clinical study was approved by the Ethical Committee of Medical University in Plovdiv, Bulgaria. The study is sponsored by Scientific Research Project No. Ho - 04 - 2018, Medical University of Plovdiv-Bulgaria. 63 teeth scheduled for extraction were selected in 60 patients ( 31 female patients and 29 male patients, mean age $42.21 \pm 13.7)$ All patients were consulted for the need of surgical extraction and implant placement procedure and signed an informant consent.

The inclusion criteria were as follows:

- presence of tooth with an indication for extraction

- presence of adjacent teeth

$-\geq 18$ years of age

- ASA (Physical Status Classification System, American Society of Anesthesiologist) I (normal healthy patient) or II (patient with a mild systemic disease)

- good oral hygiene

The exclusion criteria included:

- ASA III or IV patients

- uncontrolled diabetes

- smokers ( $\geq$ than 10 cigarettes/day)

- use of immunosuppressant medication, use of anticoagulants

- adjacent tooth extractions

- acute odontogenic infection of the tooth to be extracted or a diffuse infectious process next to the site to be intervened.

\section{Surgical treatment:}

After administering adequate local anesthesia, an intrasulcular incision around the tooth to be extracted was followed by two vertical diverging incisions reaching the mesial/distal papilla of the adjacent teeth, and a mucoperiosteal flap was elevated both from the buccal and oral site. (Fig. $1 \mathrm{a}, \mathrm{b})$ The tooth was extracted as a traumatically as possible. (Fig. 1 c) The post-extraction socket was carefully debrided and filled with graft material (PlateletRich Fibrin-PRF or BoneAlbumin ${ }^{\mathrm{TM}}$, OrthoSera Dental, Gyor, Hungary). (Fig. 2 a, b) In the control group, the postextraction socket was left with a blood clot only. (Fig. 2 c) Following a periosteal slitting (Fig.1 d), the mucoperiosteal flap was adapted without tension in order to obtain primary closure of the socket, and it was sutured with 0000 nonresorbable thread.Four months after the socket preservation procedure a surgical reentry was performed in order to place the dental implant. Incisions were performed in the same way as when performing the socket preservation procedure. After elevation of the mucoperiosteal flap, a $2.5 \mathrm{~mm}$ trephine burr was used as a pilot drill and for bone harvesting. (Fig. 3 a, b) The diameter of the trephine burr was smaller than the final drill used in the surgical implant protocol. The Dental implant was then placed according to the manufacturer's protocols. (Fig.3 c,d) A healing cap was placed, and the mucoperiosteal flap was readapted and sutured with 0000 sutures.

Fig. 1. a) Intrasulcular and two vertical incisions extraction b) elevating the mucoperiosteal flap c) periosteal slitting d) a traumatic tooth extraction
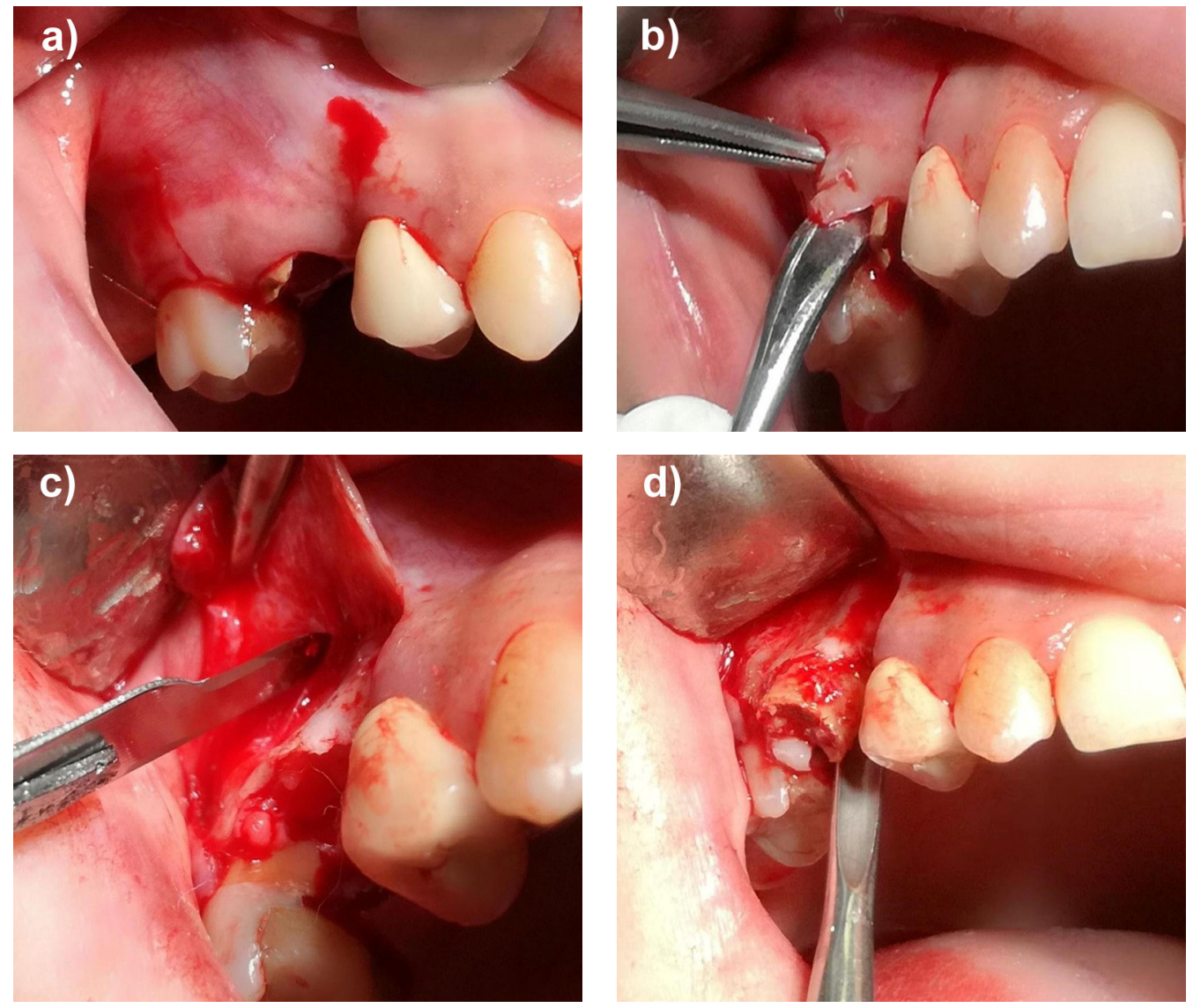
Fig. 2 a) Post exctraction socket filled with allograft b) post extraction socket filled with PRF as a sole grafting material c) post extraction socket from the control group - blood clot only
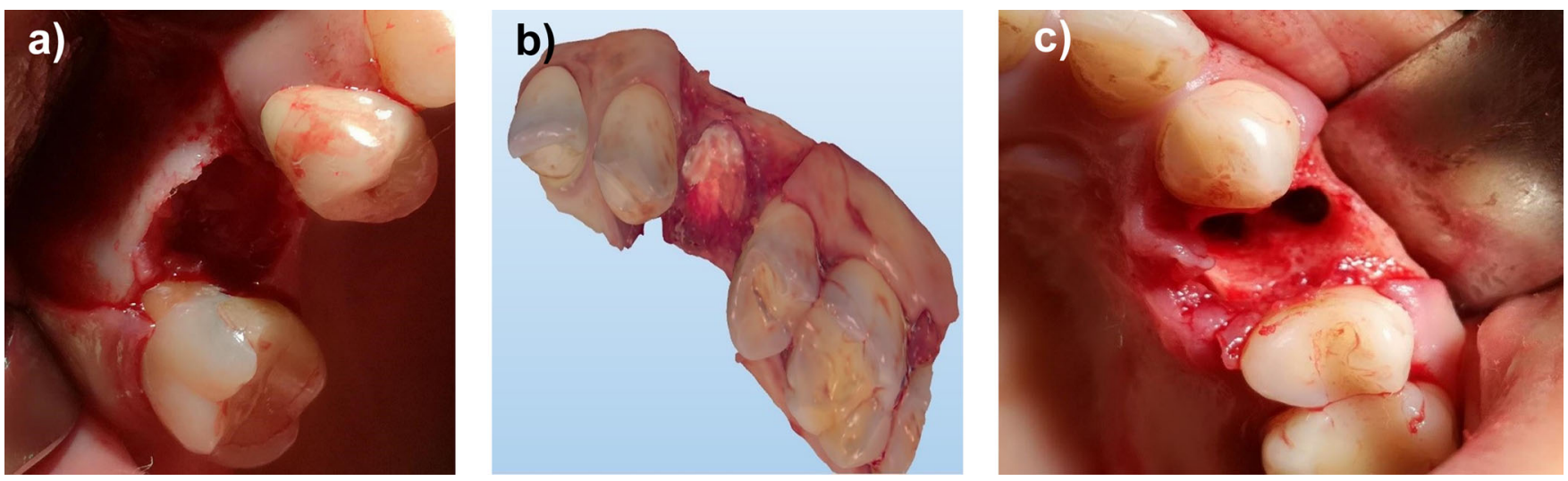

Fig. 3. a) Bone harvesting with trephine drill b) Shaped bone profile for the dental implant c) placement of the dental implant d) placed dental implant
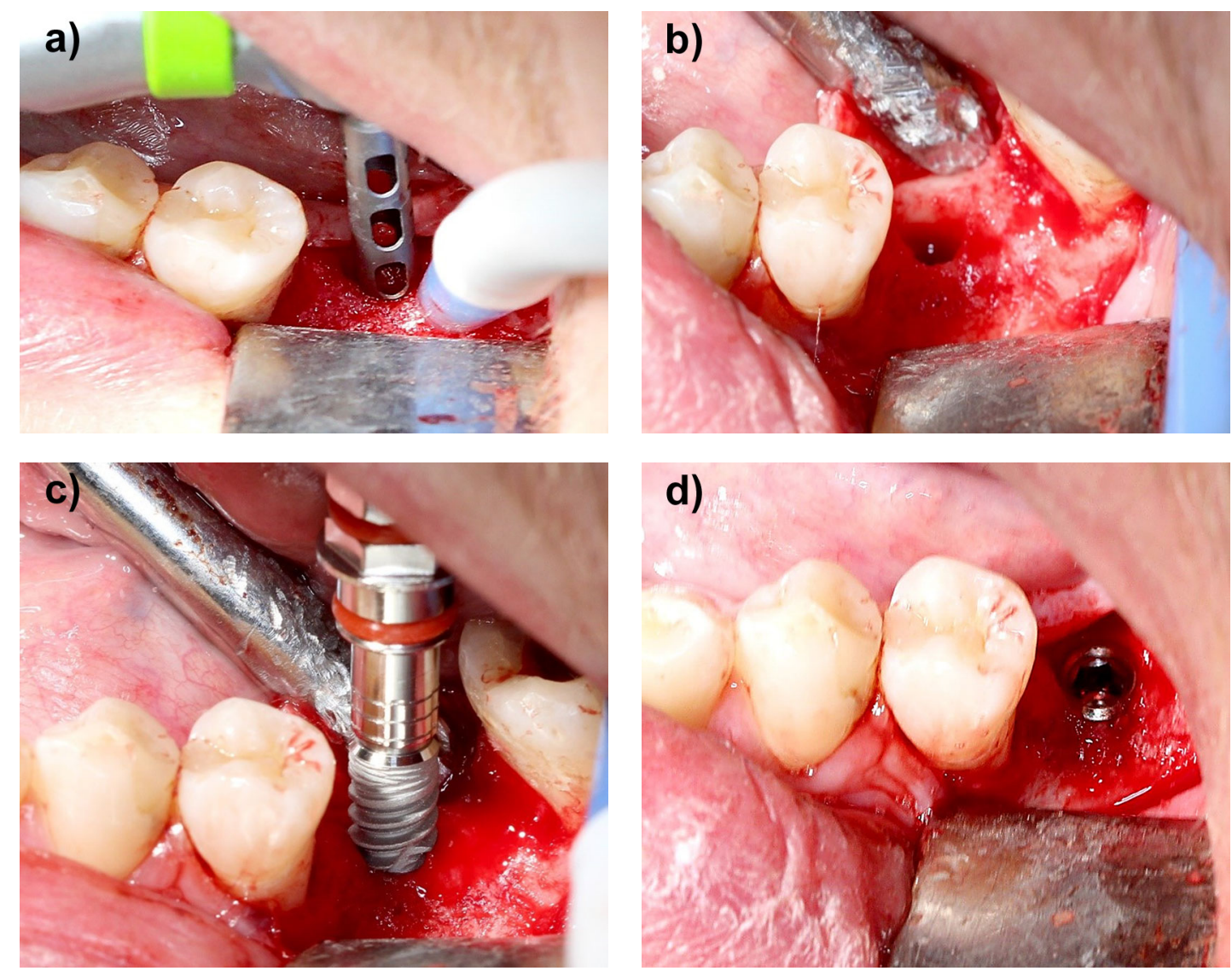

\section{Intraoral scanner Trios:}

In our clinical study, Trios intraoral scanner is used for capturing the operating area in two stages.

- In stage one, we scan the postextraction socket, including bone walls and adjacent teeth immediately after placement of the grafting material. In the control group intraoral scanner is performed without placement of substitute material inside the socket.

- Stage two is performed 4 months after the socket preservation procedure. Intraoral scans were performed immediately after elevating the mucoperiosteal flap. The virtual model in the second stage also contains the bone struc-

ture, which has formed during the healing period, including buccal and oral bone wall and adjacent teeth.

\section{Preparation of PRF:}

After the venipuncture of v. cubity with a $10 \mathrm{ml}$ vacuum test-tube (Advanced-PRFTM), 9ml of blood was taken from the patient. The blood was then immediately put into a PRF DUO (Process for $\mathrm{PRF}^{\circledR}{ }_{\text {-France) }}$ centrifuge for 8 minutes at $1300 \mathrm{rpm}$. The A- PRF membrane was formed out of two A-PRF clots by putting them in a metal box - A-PRF Box ${ }^{\circledR}$. (Fig. 4a, b) 
Fig. 4. a) Separation of the Fibrin clot from erythrocytes b) PRF membranes placed in A-PRF Box

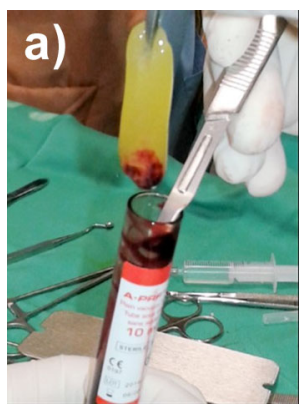

Postoperative care:

Postoperatively patients were prescribed antibiotic both after socket preservation and after implant placement procedures (Amoxicillin 1000mg every 12 hours for 5 days) and non-steroid anti-inflammatory drugs (Nimesulide $100 \mathrm{mg}$ ). Patients were instructed to rinse with $0.12 \%$ chlorhexidine twice a day for 2 weeks. Sutures were removed after 10 days.

\section{Histomorphometric analysis:}

The bone biopsies were fixed in 10\% neutral buffered formalin. Each specimen was examined for vital bone, connective tissue and residual bone/other fractions under 20x minimal magnification (Fig. 5) The material is dehydrated and enlightened with xylene. Bone fragments are incorporated into a paraffin block, cut on a rotational basis into multiple sections (3-4 microns) and stained with Hematoxylin - Eosin for light microscopic observations. Digital images were imported into image analysis software (ImageJ) to calculate the percentage of newly formed bone, connective tissue and residual bone graft particles.

\section{Three-dimensional virtual models evaluation:}

The three-dimensional replications of the field of interest during the surgical manipulation during socket preservation and 4 months after being in "stl." Format. (Fig. 6 a, b) The virtual models are imported into the three-dimensional processing software - "Meshlab" (ISTI - CNR Rome Italy). In the program models are superimposed with each other with the function - "Align". Models are aligned to each other according to the surfaces or points that have not undergone alterations during the post-operative period the adjacent teeth. (Fig. 7 a,b)
Bone resorption is evaluated by measuring the distance between different areas of the aligned models. This is performed by using the "Compute planar section" function in the menu - "Filters> Geometric measurements and computations". Cutting slices through the aligned virtual models at $0.5 \mathrm{~mm}$ each were made. (Fig. 8) Each cut indicates the outer contours of each stl. model. In order to measure the line spacing between the two outer contours of the models, the "measure tool" was applied. Two horizontal distances buccal and oral and here vertical distances - buccal, oral and central were measured. The mean result of the summarized horizontal distances in all the cutting slices revealed the mean horizontal resorption, and the mean result of the vertical distances showed the vertical resorption value.

Fig. 5. Light microscopic analysis 20x minimal magnification. VB - vital bone; CT- connective tissue; $\mathrm{RG}-$ residual graft/immature bone

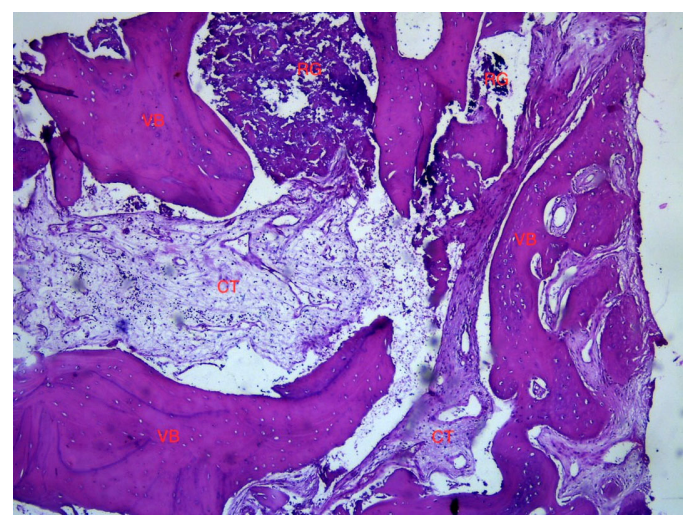

Fig. 6. Virtual models of the surgical field a) during socket preservation b) 4 months after
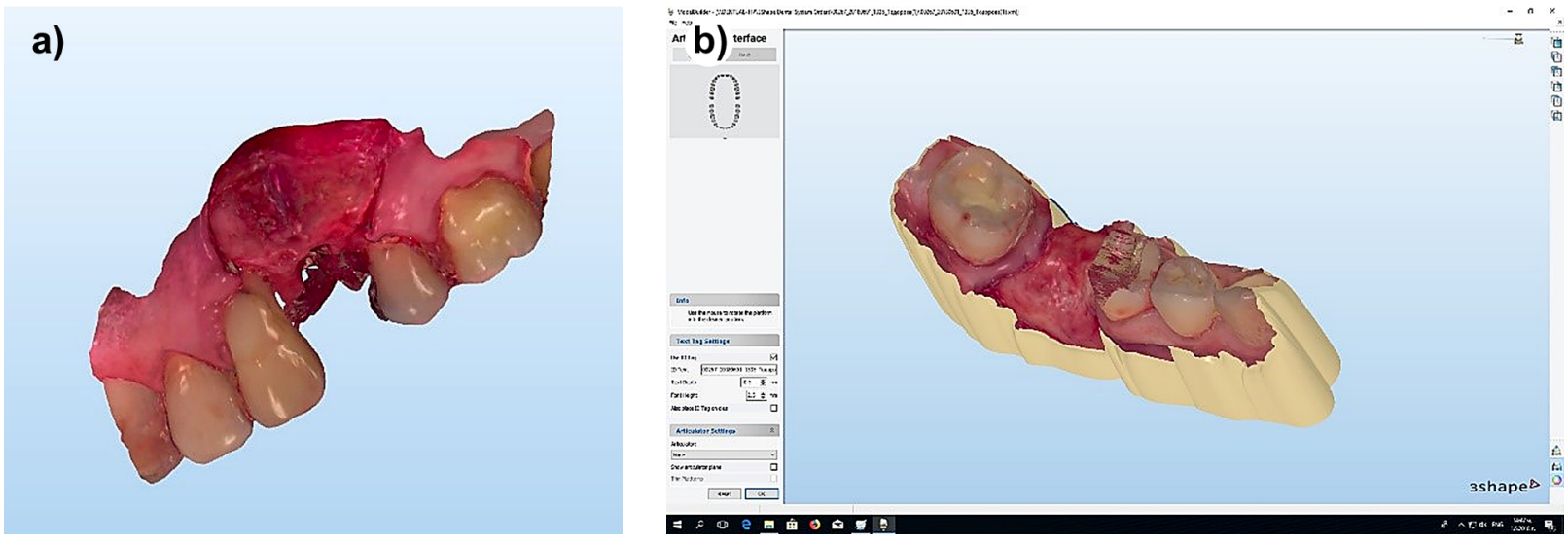
Fig. 7. Alignment of the virtual models

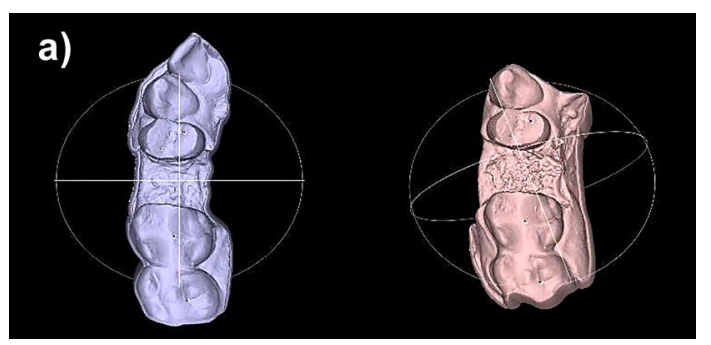

Fig. 8. Measuring the line spacing between the two outer contours of the superimposed models

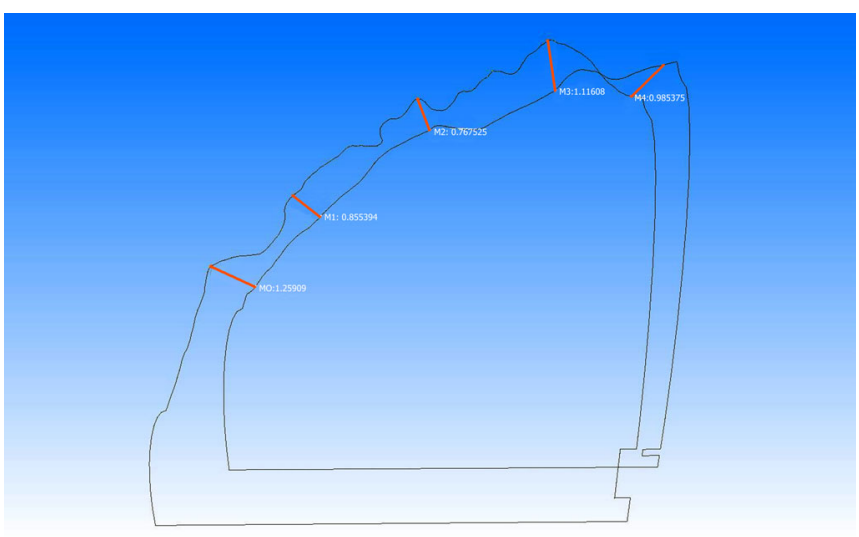

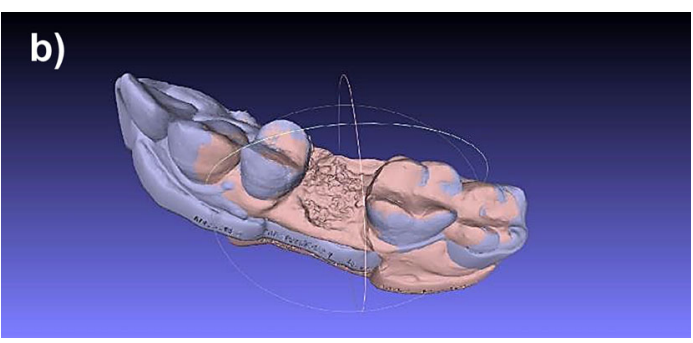

\section{RESULTS}

Virtual models analysis:

- Vertical bone resorption

In both of the grafted areas results for vertical bone resorption reveal close values with no significant difference allograft $(0.87 \pm 0.21)$ and PRF $(0.91 \pm 0.24)$. The control group showed significantly higher values for mean arithmetic and standard deviation - control group (1.38 \pm 0.14$)$. The OneWay ANOVA analysis revealed a significant difference of the vertical bone resorption values between both the tested groups and the control group. $[\mathrm{F}(2.49)=17.54$, $\mathrm{P}<0.001]$. The Tukey follow-up test revealed significantly lower vertical resorption values in the groups preserved either with allograft or PRF as a sole grafting material. (Diagram 1).

Diagram 1. Vertical bone resorption depending on the material used for socket preservation

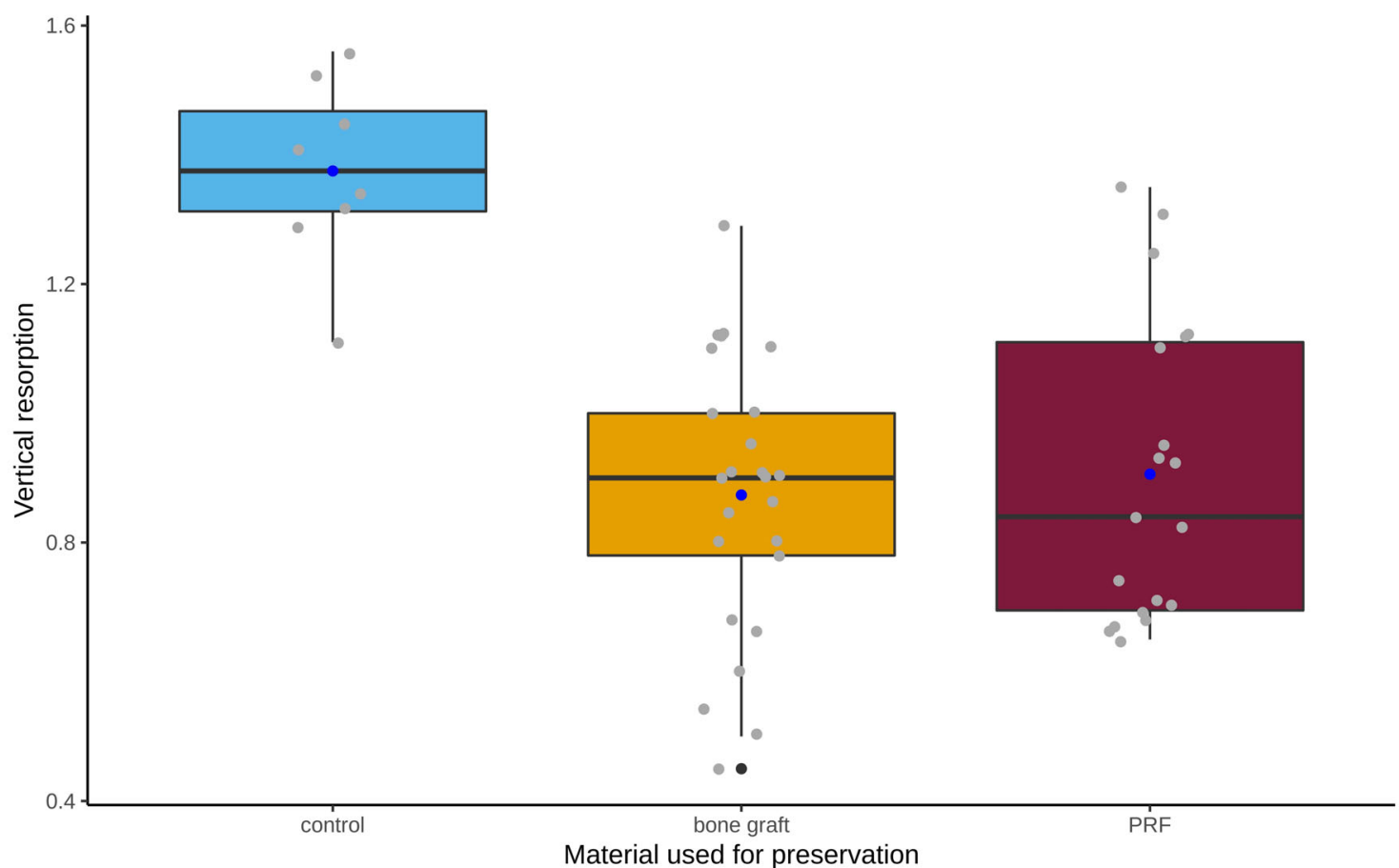

- Horizontal bone resorption

In both of the grafted areas results for vertical bone resorption reveal close values with no significant difference allograft (1.33 \pm 0.25$)$ and PRF (1.52 \pm 0.31$)$ The control group showed significantly higher values for mean arithmetic and standard deviation - control group $(2.39 \pm 0.4)$. The OneWay
ANOVA analysis revealed a significant difference of the vertical bone resorption values between both the tested groups and the control group. $[\mathrm{F}(2,49)=17.54, \mathrm{P}<0.001]$. The Tukey follow-up test revealed significantly lower horizontal resorption values in the groups preserved either with allograft or PRF as a sole grafting material. (Diagram 2). 
Diagram 2. Vertical bone resorption depending on the material used for socket preservation

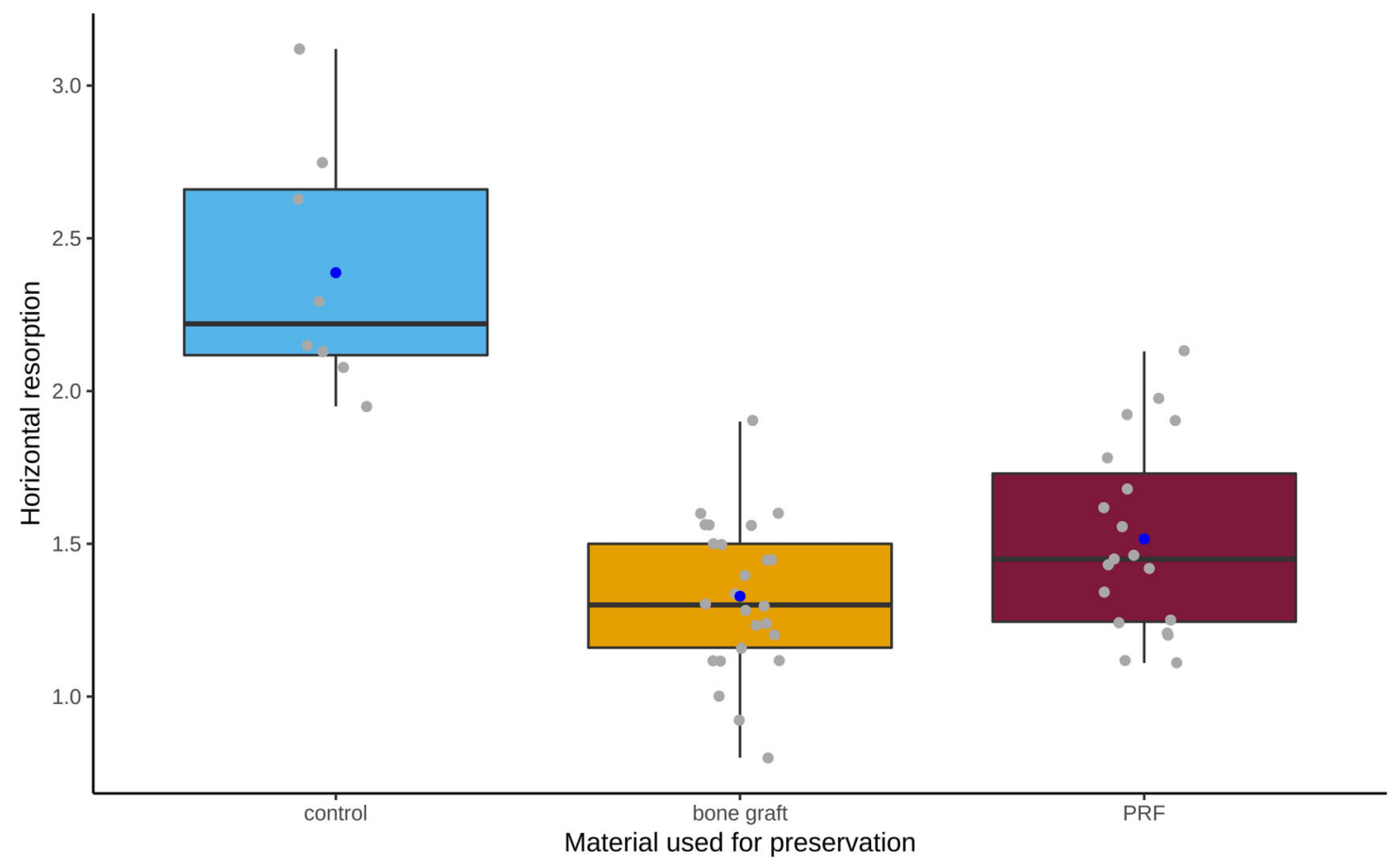

\section{Histomorphometric analysis}

- Vital bone formation

In both of the grafted areas results for vital bone formation reveal close values with no significant difference allograft $(65.92 \pm 10.91 \%)$ and PRF $(60.48 \pm 9.88 \%)$. The

control group showed significantly lower values for newly formed bone percentage - control group $(36.93 \pm 14.94 \%)$. (Diagram 3)

Diagram 3. Percentage of vital bone formation in the different groups

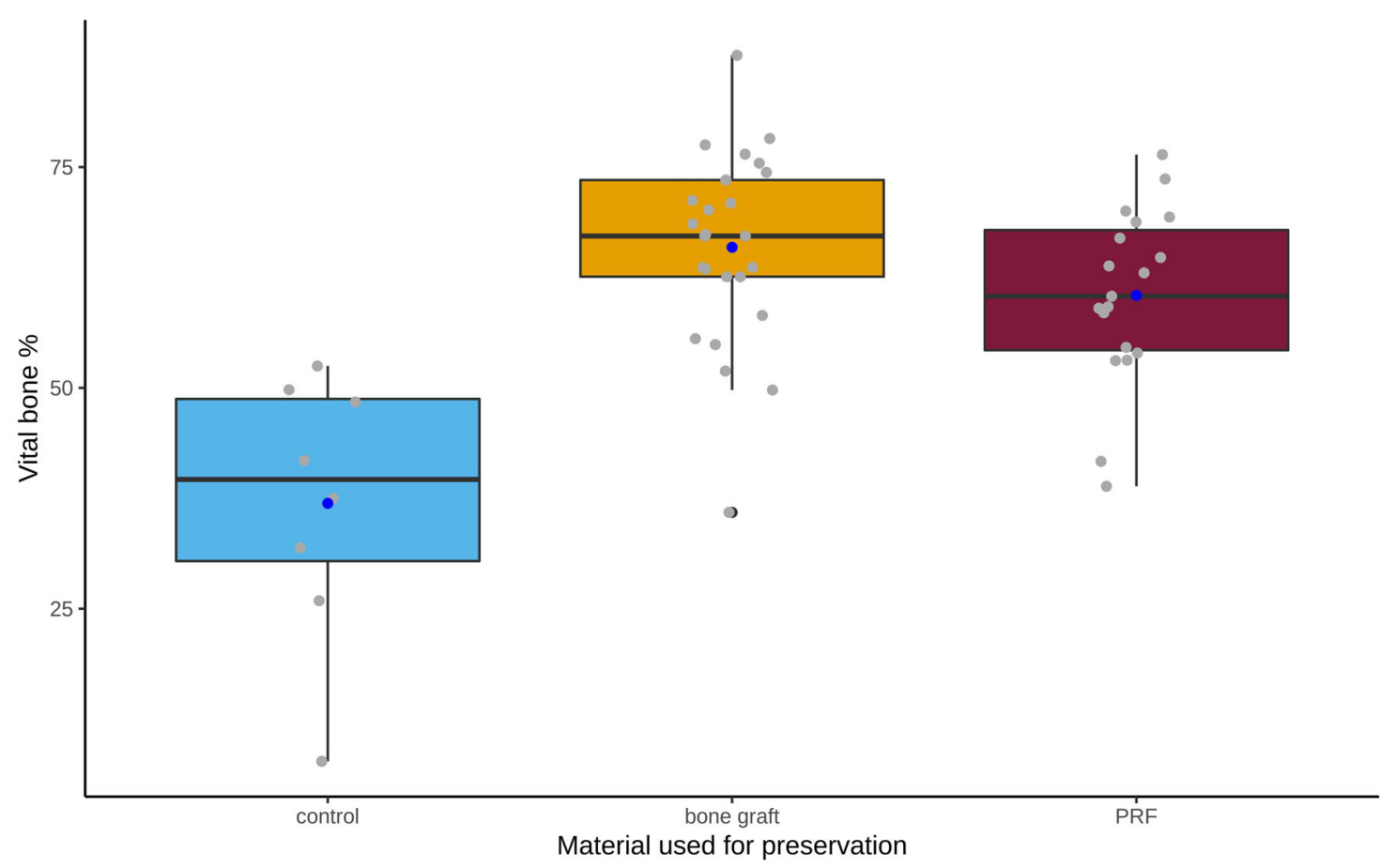


- Connective tissue

In both of the grafted areas results for the percentage of connective tissue reveal close values with no significant difference allograft $(24.37 \pm 9.35 \%)$ and PRF
$(28.53 \pm 8.66 \%)$. The control group showed significantly higher values for percentage of connective tissue - control group $(53.7 \pm 17.79 \%)$. (Diagram 4$)$

Diagram 4. Percentage of connective tissue in the different groups

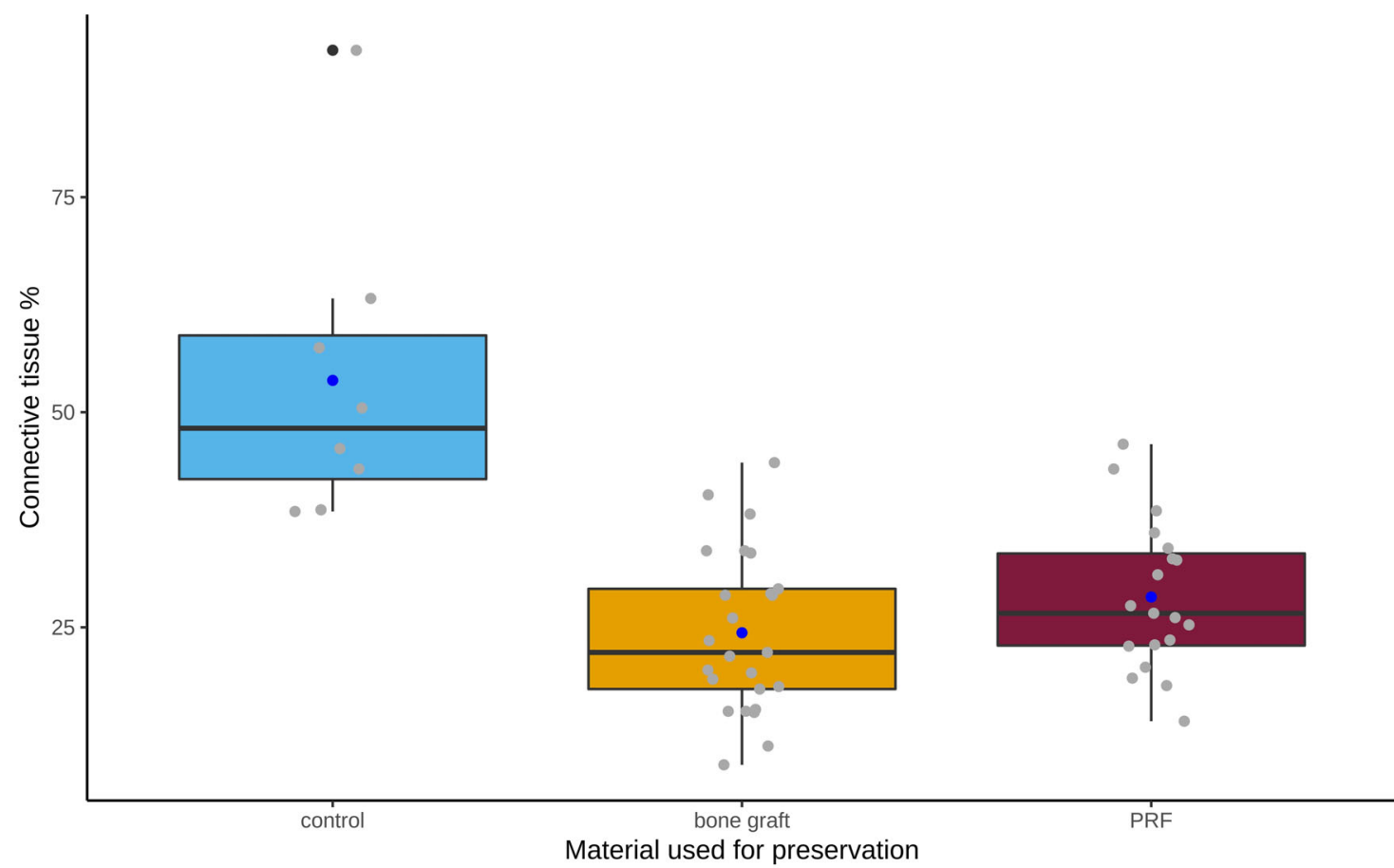

- Residual graft particles

Mean values for the quantity of residual bone for- all of the groups - allograft $(9.59 \pm 5.38 \%)$, PRF (10.99 \pm mation/woven bone are with no significant difference in $6.39 \%)$ and control group $(9.36 \pm 6.49 \%)$. (Diagram 5)

Diagram 5. Percentage of residual graft particles in the different groups

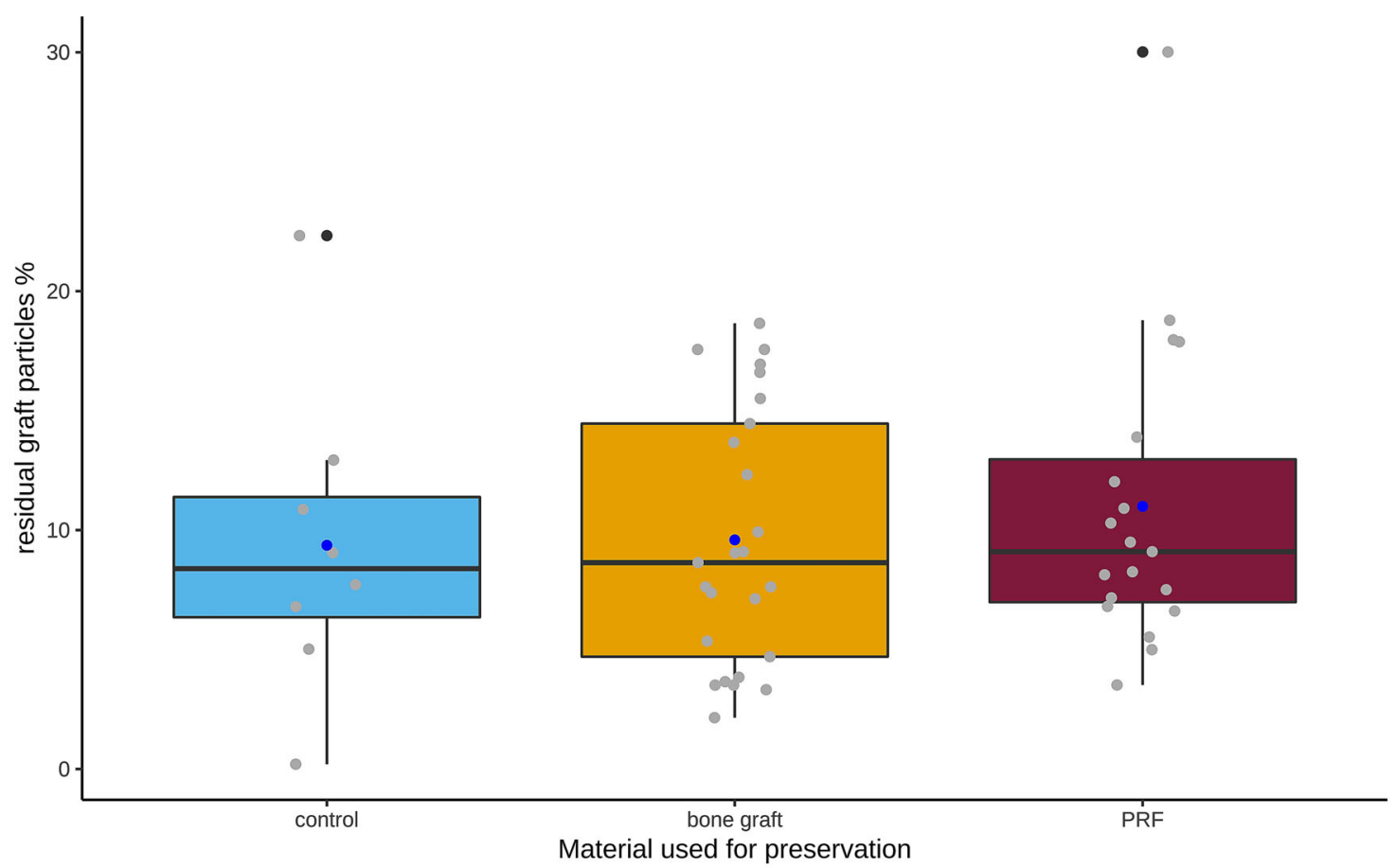




\section{DISCUSSION:}

The present randomized clinical trial aims to compare dimensional ridge alterations and histomorphometric data following tooth extraction and socket preservation with freeze dried bone allograft (BoneAlbumin ${ }^{\mathrm{TM}}$, OrthoSera Dental, Hungary) and PRF.

After tooth extraction and socket preservation procedure, horizontal resorption is always more pronounced than the vertical [11]. Tan et al.[2] have made a systematic review of the existing literature for assessing changes in both hard and soft tissues of the alveolar ridge until 12 months after tooth extraction in humans. The authors conclude that the studies show a horizontal bone loss of $29 \%$ to $63 \%$ and a vertical bone loss of $11 \%$ to $22 \% 6$ months after teeth extraction. These studies show a rapid decrease in bone volume over the first 3 to 6 months, followed by a gradual decrease in the rate of resorptive changes. Temmerman et al. [12] performed a socket preservation procedure on 22 patients by randomly dividing them into two groups - socket preservation with PRF as a sole grafting material and control group with a blood clot only. The mean values for vertical and horizontal resorption 3 months after the procedure are close to the results obtained in our study. Clark et al. [13] conducted a randomized clinical study to compare the effect of PRF and allograft after socket preservation. They randomly allocated 40 patients in 4 groups, with the first group performing an alveolar preservation procedure with A-PRF, the second with A-PRF + FDBA, the third with FDBA, the fourth group was a control group in the study. The results revealed that the test groups in the study (A-PRF and A-PRF + FDBA) show significantly less vertical resorption than the control group. In another study by Samandari et al. [14] performed on dogs, there was no significant difference in bone resorption after socket preservation with FDBA and with FDBA + PRGF. The results for vertical and horizontal bone resorption after socket preservation in our clinical study correlate with the results presented in the scientific literature. The results confirm that socket preservation with bone substitutes or biologically active substances as well as the combination of both significantly reduces the rapid processes of resorption of alveolar bone during the first months after tooth extraction. In comparison, the post-extraction socket filled with only a blood clot undergoes significantly greater resorptive changes in the horizontal and vertical direction.

In our available scientific literature, we have not found in vivo measurement of bone resorption using the Trios intraoral scanner (3Shape, Copenhagen, Denmark). The amount of vertical and horizontal bone resorption has been investigated with a variety of methods. Researchers rely on two basic assessment techniques - clinical and radiographic. They range from a clinical assessment with plastic models or individually made acrylic bands, radiographic assessment, or histological investigations on experimental animal models [15]. Despite the fact that the abovementioned methods are validated in a number of scientific publications, they have several disadvantages: they are prone to errors due to technical and human constraints; clinical methods take longer and are more prone to measurement errors [16]; the patient is subjected to further X-ray exposure, and most of them increase treatment / research costs [17]. In the present study, an intraoral scanner Trios (3Shape, Copenhagen, Denmark) was used. Our concept of using a 3D intraoral bone resorption scanner is based on its unprecedented accuracy of fewer than 20 micrometres and precision of fewer than 10 micrometres, and the ability to obtain very accurate $3 \mathrm{D}$ virtual models of the bone structures in vivo. [18]

In the present study, averaged percentages of the newly formed bone after socket preservation with PRF as a sole graft material $(60.48 \%)$ were significantly higher than the mean values for the control group $(36.93 \%)$. These findings suggest that the growth factors contained in the PRF may play a role in promoting bone regeneration over a prolonged period of time. Diss et al. [19] in a one-year prospective sine mucosal elevation study using osteotomes and PRF demonstrated that the PRF fibrin matrix directly stimulates angiogenesis. PRF has an osteonecrotic and / or osteoinductive property that stimulates bone regeneration[20]. In their study, Choukroun et al.[20] used FDBA and PRF for a lateral sinus lift to augment bone volume. The authors divide patients into two groups - control - sinus lift with FDBA and test group - sinus lift with FDBA and PRF. Histomorphometric analysis showed that the bone structures between the control group (FDBA only) and the test group (FDBA + PRF) appeared to be similar. These data correlate with the results of our study (mean amount of newly formed bone with the preservation of the alveolus with PRF - $60.48 \%$ and the average amount of newly formed bone after allograft contention (FDBA) - 65.92\%).

\section{CONCLUSION:}

The study reveals that A-PRF as a sole grafting material or freeze-dried bone allograft in combination with PRF membrane are both suitable for ridge preservation procedures. The study demonstrates a novel in vivo method (Trios intraoral scanner) for measuring bone resorption after ridge augmentation procedures. This method is more accurate and precise compared to other well-established methods for investigation bone dimensional changes. More research is needed for verification of the method.

\section{REFERENCES:}

1. Hauser F, Gaydarov N, Badoud I, Vazquez L, Bernard JP, Ammann P. Clinical and Histological Evaluation of Postextraction Platelet-rich Fibrin Socket Filling: A Prospective Randomized Controlled Study. Implant Dent. 2013 Jun;22(3):295-303. [PubMed]

\section{[Crossref]}

2. Tan WL, Wong TL, Wong MC, Lang NP. A systematic review of postextractional alveolar hard and soft tissue dimensional changes in humans. Clin Oral Implants Res. 2012 Feb;23 Suppl 5:1-21. [PubMed] [Crossref]
3. Iasella JM, Greenwell H, Miller RL, Hill M, Drisko C, Bohra AA, et al. Ridge preservation with freeze-dried bone allograft and a collagen membrane compared to extraction alone for implant site development: a clinical and histologic study in humans. $J$. 
Periodontol. 2003 Jul;74(7):990-9. [PubMed] [Crossref]

4. Sadeghi R, Babaei M, Miremadi SA, Abbas FM. A randomized controlled evaluation of alveolar ridge preservation following tooth extraction using deproteinized bovine bone mineral and demineralized freeze-dried bone allograft. Dent Res J (Isfahan). 2016 Mar-Apr;13(2):151-9. [PubMed] [Crossef]

5. Piattelli A, Scarano A, Corigliano M, Piattelli M. Comparison of bone regeneration with the use of mineralized and demineralized freeze-dried bone allografts: a histological and histochemical study in man. Biomaterials. 1996 Jun;17(11):1127-31. [PubMed] [Crossref]

6. Dohan DM, Choukroun J, Diss A, Dohan SL, Dohan AJ, Mouhyi J, et al. Platelet-rich fibrin (PRF): a secondgeneration platelet concentrate. Part II: platelet-related biologic features. Oral Surg Oral Med Oral Pathol Oral Radiol Endod. 2006 Mar;101(3):e4550. [ubMed] [Crossref]

7. Srinivas B, Das P, Rana MM, Qureshi AQ, Vaidya KC, Ahmed Raziuddin SJ. Wound Healing and Bone Regeneration in Postextraction Sockets with and without Platelet-rich Fibrin. Ann Maxillofac Surg. 2018 JanJun;8(1):28-34. [PubMed] [Crossref]

8. Geurs N, Ntounis A, Vassilopoulos P, Van der Velden U, Loos BG, Reddy M. Using growth factors in human extraction sockets: a histologic and histomorphometric evaluation of shortterm healing. Int J Oral Maxillofac Implants. 2014 Mar-Apr;29(2):485-96.

\section{[PubMed] [Crossref]}

9. Horvath A, Mardas N, Mezzomo LA, Needleman IG, Donos N. Alveolar ridge preservation. A systematic review. Clin Oral Investig. 2013 Mar; 17(2):341-63. [PubMed] [Crossref]

10. Wood RA, Mealey BL. Histologic comparison of healing after tooth extraction with ridge preservation using mineralized versus demineralized freeze-dried bone allograft. $J$ Periodontol. 2012 Mar;83(3): 329-36. [PubMed] [Crossref]

11. Araujo MG, Lindhe J. Dimensional ridge alterations following tooth extraction. An experimental study in the dog. J Clin Periodontol. 2005 Feb;32(2):212-8. [PubMed] [Crossref]

12. Temmerman A, Vandessel J, Castro A, Jacobs R, Teughels W, Pinto $\mathrm{N}$, et al. The use of leucocyte and platelet-rich fibrin in socket management and ridge preservation: a split-mouth, randomized, controlled clinical trial. $J$ Clin Periodontol. 2016 Nov;43(11): 990-9. [PubMed] [Crossref]

13. Clark D, Rajendran Y, Paydar S, Ho S, Cox D, Ryder M, et al. Advanced platelet-rich fibrin and freezedried bone allograft for ridge preservation: A randomized controlled clinical trial. J Periodontol. 2018 Apr;89(4): 379-387. [PubMed] [Crossref]

14. Samandari MH, Haghighat A, Torabinia N, Taghian M, Sadri L, Naemy V. Socket preservation using freeze dried bone allograft with and without plasma rich in growth factors in dogs. Dent Res J (Isfahan).2016 Sep;13(5):432-439. [PubMed]

\section{[Crossref]}

15. Vignoletti F, Matesanz P, Rodrigo D, Figuero E, Martin C, Sanz M. Surgical protocols for ridge preservation after tooth extraction. A systematic review. Clin. Oral Implants Res. 2012 Feb;23 Suppl 5:22-38. [PubMed] [Crossref]

16. Preus HR, Torgersen GR, Koldsland OC, Hansen BF, Aass AM, Larheim TA, et al. A new digital tool for radiographic bone level measurements in longitudinal studies. BMC Oral Health. 2015; Sep 8;15:107. [PubMed] [Crossref]

17. Zlatev S, Ivanova V, Chenchev I. A Method For Volumetric Evaluation of Post-Extraction Site's Alveolar Bone Change In Human Subjects. IOSRJDMS. 2017; 16(11):76-78.

18. Hack GD, Patzelt SBM. Evaluation of the Accuracy of Six Intraoral Scanning. Am Dent Assoc. 2015; 10(4):1-5

19. Diss A, Dohan DM, Mouhyi J, Mahler P. Osteotome sinus floor elevation using Choukroun's platelet-rich fibrin as grafting material/ : a 1-year prospective pilot study with microthreaded implants. Oral Surg Oral Med Oral Pathol Oral Radiol Endod. 2008 May;105(5):572-9. [PubMed] [Crossref]

20. Choukroun J, Diss A, Simonpieri A, Girard MO, Schoeffler C, Dohan SL, et al. Platelet-rich fibrin (PRF): A second-generation platelet concentrate. Part IV: Clinical effects on tissue healing. Oral Surg Oral Med Oral Pathol Oral Radiol Endod. 2006 Mar;101(3):56-60. [PubMed]

Please cite this article as: Ivanova V, Chenchev I, Zlatev S, Kanazirski N. Dimensional Ridge Alterations and Histomorphometric Analysis Following Socket Preservation with PRF or Allograft. Randomized Controlled Clinical Study. $J$ of IMAB. 2019 Oct-Dec;25(4):2853-2861. DOI: https://doi.org/10.5272/jimab.2019254.2853

Received: 19/07/2019; Published online: 20/12/2019

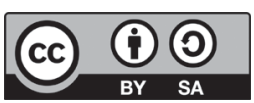

Address for correspondence:

Dr. Vasilena Ivanova - Assistant professor

Department of Oral Surgery, Faculty of Dental Medicine, Medical University Plovdiv, Bulgaria

E-mail: vasilena.v.ivanova@gmail.com 